\title{
Going Remote-Demonstration and Evaluation of Remote Technology Delivery and Usability Assessment With Older Adults: Survey Study
}

Jordan R Hill ${ }^{1}$, MSIE, PhD; Addison B Harrington ${ }^{2}$, BS; Philip Adeoye ${ }^{2}$, MS; Noll L Campbell ${ }^{2,3}$, MS, PharmD; Richard J Holden ${ }^{1,2}$, PhD

\footnotetext{
${ }^{1}$ Department of Medicine, Indiana University School of Medicine, Indianapolis, IN, United States

${ }^{2}$ Center for Aging Research, Regenstrief Institute, Indianapolis, IN, United States

${ }^{3}$ Department of Pharmacy Practice, College of Pharmacy, Purdue University, West Lafayette, IN, United States
}

\section{Corresponding Author:}

Jordan R Hill, MSIE, PhD

Department of Medicine

Indiana University School of Medicine

$1101 \mathrm{~W} 10$ th St

Indianapolis, IN, 46202

United States

Phone: 17655438559

Email: jrh6@iu.edu

\section{Abstract}

Background: The COVID-19 pandemic necessitated "going remote" with the delivery, support, and assessment of a study intervention targeting older adults enrolled in a clinical trial. While remotely delivering and assessing technology is not new, there are few methods available in the literature that are proven to be effective with diverse populations, and none for older adults specifically. Older adults comprise a diverse population, including in terms of their experience with and access to technology, making this a challenging endeavor.

Objective: Our objective was to remotely deliver and conduct usability testing for a mobile health (mHealth) technology intervention for older adult participants enrolled in a clinical trial of the technology. This paper describes the methodology used, its successes, and its limitations.

Methods: We developed a conceptual model for remote operations, called the Framework for Agile and Remote Operations (FAR Ops), that combined the general requirements for spaceflight operations with Agile project management processes to quickly respond to this challenge. Using this framework, we iteratively created care packages that differed in their contents based on participant needs and were sent to study participants to deliver the study intervention-a medication management app-and assess its usability. Usability data were collected using the System Usability Scale (SUS) and a novel usability questionnaire developed to collect more in-depth data.

Results: In the first 6 months of the project, we successfully delivered 21 care packages. We successfully designed and deployed a minimum viable product in less than 6 weeks, generally maintained a 2-week sprint cycle, and achieved a $40 \%$ to $50 \%$ return rate for both usability assessment instruments. We hypothesize that lack of engagement due to the pandemic and our use of asynchronous communication channels contributed to the return rate of usability assessments being lower than desired. We also provide general recommendations for performing remote usability testing with diverse populations based on the results of our work, including implementing screen sharing capabilities when possible, and determining participant preference for phone or email communications.

Conclusions: The FAR Ops model allowed our team to adopt remote operations for our mHealth trial in response to interruptions from the COVID-19 pandemic. This approach can be useful for other research or practice-based projects under similar circumstances or to improve efficiency, cost, effectiveness, and participant diversity in general. In addition to offering a replicable approach, this paper tells the often-untold story of practical challenges faced by mHealth projects and practical strategies used to address them.

Trial Registration: ClinicalTrials.gov NCT04121858; https://clinicaltrials.gov/ct2/show/NCT04121858 
(JMIR Mhealth Uhealth 2021;9(3):e26702) doi: 10.2196/26702

\section{KEYWORDS}

COVID-19; mobile usability testing; usability inspection; methods; aging; agile; mobile phone

\section{Introduction}

\section{Overview}

Technological advances have made it more commonplace and easier for individuals to complete tasks remotely, from online banking to visiting a doctor to paid work from home [1]. The COVID-19 pandemic revealed that remote interaction is also sometimes necessary. The need to "go remote" during the pandemic likely both accelerated innovation in telecommunication and normalized available but underused internet-based services and products, such as telehealth [2], mental health apps [3], and e-commerce [4].

The pandemic also disrupted research, forcing projects to suspend operations or implement remote noncontact methods [5-7]. Paradoxically, some studies of mobile technology faced difficulties converting to entirely remote technology delivery and usability assessment, revealing opportunities for technology projects to experiment with remote operations [8].

This paper reports our experiences in "going remote" to rapidly restart a suspended clinical trial of a mobile health (mHealth) app with older adults. We present our protocol changes, Agile project management approach, and findings from this restart project. Our experiences elucidate the opportunities and challenges of conducting technology research remotely and may inform other attempts at rapid operational change.

\section{Background}

Remote delivery and usability assessment of technology is not new $[9,10]$ but have heretofore been largely used with younger, highly educated, technologically experienced, and more homogeneous individuals, for example, college students $[9,11]$ or those using the technology as a part of their employment [12-14]. Only one study was found to employ remote methods with older adults and these were supplemented with in-person sessions for initial training and instruction [15].

Nevertheless, existing remote methods provide a foundation for technology development projects with older, disabled, or other diverse populations in which experience is limited. Potentially useful existing methods to access and evaluate technology include online questionnaires, online forums, self-kept diaries, email, telephone calls, and postal delivery [11,15-17]. More recently, videoconferencing tools are used to replicate in-person, moderated usability testing $[8,11,17]$.

These methods have, to our knowledge, not been adapted for fully remote projects with older adult users, although some work has been done with younger and older adults with various disabilities [18]. This gap is relevant because older adults are the fastest growing population of technology users [19] but also one of the most diverse populations in terms of their motivations to use technology and their physical, cognitive, and sensory capabilities [20]. Additionally, among older adults, factors such as race and ethnicity, income, literacy, education level, age group (eg, <75 vs $\geq 75$ years of age), and community characteristics (eg, rural vs urban) significantly impact access and use of the internet and other technologies [21-26]. Thus, one challenge of "going remote" with older adults is to accommodate between-age and within-age diversity.

Accordingly, prior research has suggested best practices and adjustments for in-person technology assessment with older adults. For example, because think-aloud procedures and psychometric scales (eg, Likert) may not work well for this population, it may be better to engage older adults one-on-one in a guided interview to elicit information [27,28]. Others recommend personalizing usability testing protocols with attention to fidelity in those with cognitive decline or disabilities [29].

\section{Objective}

The objective of the reported project, Project C (COVID), was to remotely deliver and conduct usability testing for an $m$ Health technology intervention for older adult participants enrolled in a clinical trial of the technology. The need for remote delivery arose from restrictions on in-person research activities due to a global pandemic and an ethical obligation to reduce risk for older adult participants and study personnel. Meeting the objective required special effort due to participants' diverse technology ownership, experience, motivations, and skill levels. For example, a subset of participants did not have a smartphone or home internet and required the study to provide these. Thus, the team could not simply provide an online link to the app for all participants to download. Below, we describe how we developed these remote operations to deliver and test mHealth technology to a heterogenous older adult population participating in human subjects research during a pandemic.

\section{Methods}

\section{Overview}

Project $\mathrm{C}$ occurred in the context of the Brain Safe randomized clinical trial, described in depth elsewhere [30]. The study enrolled adults aged 60 years or older in Indiana, USA, who were using medications with anticholinergic effects that may increase risk to brain health for older adults. In the trial's parallel treatment arms, participants received a mobile app to use for 12 months, either the Brain Safe intervention app or the Med Safe attention control app. Both were native Android or iOS apps, Health Insurance Portability and Accountability Act (HIPAA) compliant, and optimized for smartphones, with the following features:

1. Self-registration.

2. Passwordless log-in, via verification token for each log-in.

3. Four-tab navigation.

4. A medication list created by the user through searching and browsing, selecting medication choices, and numeric data entry. 
5. Monthly reminders to complete medication review.

6. Text and video educational materials.

7. PDF report output and sharing.

8. A score calculator.

Whereas the score calculator in the Med Safe app merely calculated the total number of medications, the Brain Safe app calculated a brain harm risk score, let users simulate the effect on risk of adding or removing anticholinergic medications, showed alternative treatments, and helped users start a conversation with their health care professionals about medication safety. Additional detail on features, design, development, and prior testing are available elsewhere [30,31].

Both apps were designed to be loaded on a smartphone meeting minimum hardware, software, and connectivity requirements. Originally, standard operating procedures in the trial were to conduct installation, account registration, training, first-time use, initial technical support, troubleshooting, and usability observations in person at the participant's home or in a research office, with continuing opportunities for remote or in-person support. A smartphone, typically a 5.8-inch Samsung Galaxy S9 or S10, with voice calling and unlimited text and data plans was provided to any participant needing it for the study's duration.

Project C was launched on March 13, 2020, when in-person trial operations were suspended due to COVID-19-related institutional policies, city and state governmental mandates, and an ethical imperative to reduce risk to participants, study personnel, and the community. At the time, the study had consented and enrolled 56 participants out of a planned 700. Of those enrolled, 7 (13\%) had received the Brain Safe or Med Safe app and the remaining $49(88 \%)$ were the target group for which Project $\mathrm{C}$ was designed. Over time, Project $\mathrm{C}$ provided remote capability for newly enrolled participants and is still ongoing. We present results from the first 6 months of iterative development of Project C, through September 13, 2020.

All procedures in the Brain Safe trial, which was registered at ClinicalTrials.gov (NCT04121858), were approved by the Indiana University Institutional Review Board (IRB). Participants consented to the study, including receiving the app, app-related interactions, and usage data collection, and completed usability and technology acceptance questionnaires. Additional procedures for Project $\mathrm{C}$, for example, providing remote technical support or collecting additional feedback on usability, were conducted for the purpose of improving operations and quality; they were, therefore, not deemed human subjects research. However, changes to allow remote consenting processes with newly enrolled participants were approved as protocol amendments by the IRB.

\section{Conceptual Model}

We framed the shift to remote operations as a challenge akin to, but less extreme than, spaceflight. Spaceflight missions send people and payloads (eg, satellites, probes, and rovers) to extremely distant locations, including Mars and the International Space Station [32-34]. Mission success requires solving three challenges: (1) delivery (ie, getting payload to its destination), (2) support (ie, remotely identifying and solving problems throughout the mission), and (3) return (ie, retrieving materials or information during or after the mission) (see Figure 1, A) [35-38]. Similarly, the challenge of remote technology intervention research is three-fold: (1) delivery of the technology to a diverse set of distant recipients; (2) support of the technology and its users, including training, technical support, and communication; and (3) return of data (eg, usability assessments), equipment, and feedback regarding status (see Figure 1, B).

To this spaceflight operations model, we added an Agile project management approach [39] to address the need for innovation and timeliness; the Agile approach is represented by feedback loops in Figure 1, B. The Agile approach was introduced by software developers to promote iterative design, delivering products on a short timeline, responding to user feedback, and learning from failures [40]. Agile approaches have since been used to improve health care delivery, for example, to identify, adapt, implement, and evaluate evidence-based clinical services [41]. Holden and Boustani [42] argued that replacing status quo health care operations with an Agile Mindset could help organizations more nimbly and effectively cope with crises, including the COVID-19 pandemic. In our work, we adopted the three principles of an Agile Mindset proposed by those authors:

1. Sprints. Accelerate progress toward a minimum viable product, to be progressively tested and improved in sprints.

2. Sensors. Embed sensors to collect timely, nonjudgmental, and actionable feedback, leading to learning and redesign between sprints.

3. Safe culture. Practice a culture of psychological safety, such that teams have latitude, decision authority, and resources and, above all, feel psychologically safe to innovate, experiment, and fail. 
Figure 1. (A) General model of remote spaceflight missions; (B) Framework for Agile and Remote Operations (FAR Ops).

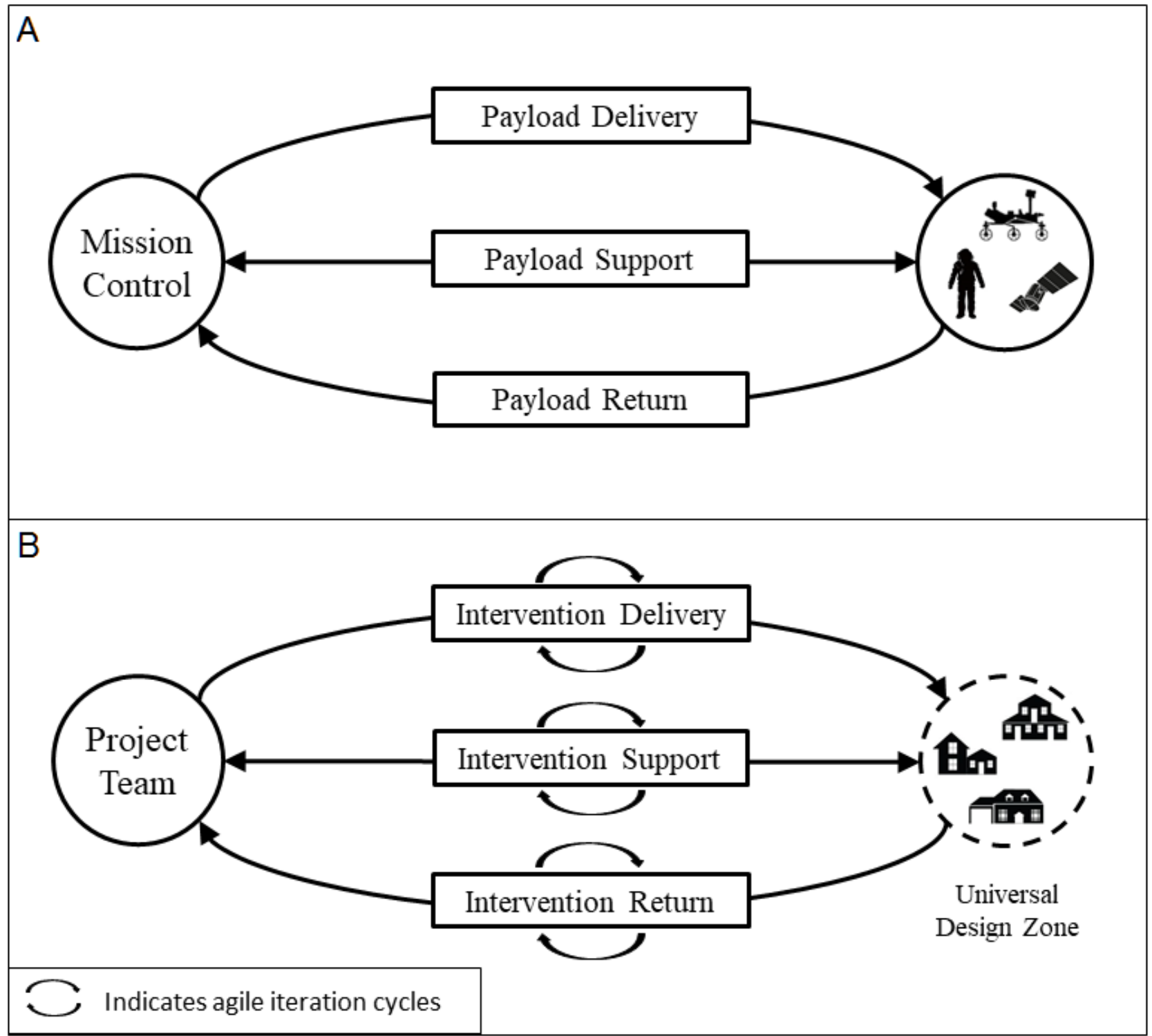

\section{Setting and Participants}

The study took place in central Indiana, USA, in partnership with Indiana University, Purdue University, Regenstrief Institute, and local not-for-profit academically affiliated health systems. Participants were consenting cognitively normal adults aged 60 years or older, English-speaking, and using prescribed anticholinergic medications; cognitive normality was determined using the six-item cognitive screener [43]. The first cohort of participants, including all described here, were receiving primary care at Indiana University Health, a statewide academic health system. Individuals were excluded if they were diagnosed with, or using medications to treat, dementia or serious mental illness; were living in an extended care facility or not managing their own medications; were unable to use an app due to sensory disability; or screened positive for cognitive dysfunction or terminal illness. All had consented to the Brain Safe trial and provided a waiver of HIPAA authorization.

\section{Procedure}

We applied the Framework for Agile and Remote Operations (FAR Ops) model to achieve technology delivery, support, and return functions in an Agile manner. A team of three study personnel formed the core Project $\mathrm{C}$ team: a part-time postdoctoral fellow, with a $\mathrm{PhD}$ in industrial and systems engineering; a part-time user experience designer and developer, with an MS in human-computer interaction; and a full-time research specialist, with a BS in neuroscience. The study principal investigator (PI) provided the team with minimal critical specifications to deliver and assess the technology. The team was expected to communicate with the PI, solicit feedback from the broader research team, and develop their own timeline, budget, and procedures. They were explicitly encouraged to be innovative and embrace failure as an opportunity to learn, but to fail early by conducting rapid cycles of testing and redesign, as described in other work on mHealth [44-46].

The team met formally once a week, maintaining informal communications on an as-needed basis, to brainstorm ideas, develop a weekly plan, and monitor the progress of solution development. They worked autonomously and communicated progress to receive feedback and support, not direction, from peers and leaders. The team determined that the first minimum viable product would be a care package containing all the 
necessary resources and information for participants to receive and use the intervention, along with a word search activity book to show goodwill and increase engagement; this token item was approved by the IRB. In the Results section, we further describe various iterations of the care package product, its delivery, and how users were supported postdelivery.

After the first minimum viable product was ready, the team followed a 2-week sprint schedule. For 1 week they remotely delivered a new batch of packages, and in the subsequent week the team collected feedback, analyzed the results, and redesigned the approach for the next week's deliveries.

To assess technology usability, the team (1) used the Simplified System Usability Scale (SUS) for Cognitively Impaired and Older Adults [47] to obtain a high-level summary usability score and (2) developed a novel usability questionnaire to obtain feedback on specific usability issues with the apps. The novel questionnaire asked for participants' general impressions of the app (eg, if they had trouble reading the text and if they were happy the app was available) as well as their impressions regarding each step of app use (opening the app, logging in, adding and removing medications, etc). Participants were also given the opportunity to express their opinions in their own words in free-text boxes. Participants were given access to an online version of the questionnaire and/or a paper version to be mailed back to the study team. The questionnaires were one way the team was able to return information from remote participants. Another method to return nonstudy data from users, deployed after the first 6 months and, therefore, not reported here, was a user and activity dashboard, with functionality to create or edit accounts remotely, monitor every log-in and in-app function use, and generate custom usage reports.

\section{Analysis}

We evaluated the performance of the project on the following criteria, as derived from Agile project management metrics:

1. Time to develop and deliver the first package (ie, minimum viable product); our goal was $\leq 6$ weeks.
2. Adherence to the 2-week sprint cycle; our goal was $100 \%$.

3. Percentage of returning usability questionnaires; no goal was set.

This approach is not textbook Agile. Instead, we have adopted a more general Agile-inspired mindset emphasizing quick progress, autonomous work, and a safe culture within the team. We decided against spending a considerable amount of time implementing more Agile metrics in favor of a quicker response. The three metrics listed above were chosen due to their simplicity and their effectiveness in demonstrating whether our approach was successful in terms of time to respond to the challenge, maintenance of an Agile Mindset, and collection of feedback.

We also analyzed and report feedback obtained from participants and team personnel to evaluate the feasibility of remote operations and the Agile approach. An in-depth analysis of collected usability results was beyond the scope of this paper.

\section{Results}

\section{Overview}

The 49 participants who were enrolled in the Brain Safe study but had not yet been delivered a study app, were the target of Project $\mathrm{C}$. The demographics of this target group are included in Table 1. No participants had significant cognitive impairments (eg, dementia).

Three main types of care packages were developed and delivered to participants; Types A, B, and C are summarized in Table 2 with the cost of each. Table 3 details the contents and methods used for each package type. Package types, and iterations within type (ie, $A_{0}$ vs $A_{1}$ ), were developed between sprints as new needs arose. For example, as some participants began receiving email instructions to download the app to their own smartphone device, the project team learned of the need to provide additional remote installation support. 
Table 1. Project C (COVID) target group demographics.

\begin{tabular}{|c|c|}
\hline Demographic & Number of participants $(\mathrm{N}=49), \mathrm{n}(\%)$ \\
\hline \multicolumn{2}{|l|}{ Sex } \\
\hline Male & $15(31)$ \\
\hline Female & $34(69)$ \\
\hline \multicolumn{2}{|l|}{ Age (years) } \\
\hline $60-64$ & $29(59)$ \\
\hline $65-69$ & $8(16)$ \\
\hline $70-74$ & $8(16)$ \\
\hline $75-79$ & $2(4)$ \\
\hline $80-84$ & $1(2)$ \\
\hline $85+$ & $1(2)$ \\
\hline \multicolumn{2}{|l|}{ Race } \\
\hline White or Caucasian & $33(67)$ \\
\hline Black or African American & $13(27)$ \\
\hline More than one race & $3(6)$ \\
\hline \multicolumn{2}{|l|}{ Education level } \\
\hline Some high school & $3(6)$ \\
\hline High school graduate or General Educational Development & $8(16)$ \\
\hline Some college & $14(29)$ \\
\hline College degree & $14(29)$ \\
\hline Master's or other advanced degree & $9(18)$ \\
\hline Other or no data & $1(2)$ \\
\hline
\end{tabular}

Table 2. Care package types.

\begin{tabular}{|c|c|c|c|c|}
\hline Type & Description & Participant needs addressed & Purpose of the package & $\begin{array}{l}\text { Cost per package } \\
\text { (US \$) }\end{array}$ \\
\hline $\mathrm{A}_{0}$ & Full package (version 1) & $\begin{array}{l}\text { For participants without their own smart- } \\
\text { phone or preferring to use a study phone }\end{array}$ & $\begin{array}{l}\text { To fulfil the technical (ie, software, hard- } \\
\text { ware, and internet) needs of participants, } \\
\text { some of whom were using a mobile app or } \\
\text { modern smartphone for the first time }\end{array}$ & $\$ 34.70$ \\
\hline $\mathrm{A}_{1}$ & Full package (version 2) & $\begin{array}{l}\text { For participants who needed fast and direct } \\
\text { delivery }\end{array}$ & $\begin{array}{l}\text { Same as } \mathrm{A}_{0} \text { and to improve the feasibility } \\
\text { and lower delivery costs }\end{array}$ & $\$ 17.70$ \\
\hline B & Interest-renewal package & $\begin{array}{l}\text { For enrolled participants who were difficult } \\
\text { to reach in other ways (ie, phone and email) }\end{array}$ & $\begin{array}{l}\text { To re-engage participants who may have } \\
\text { lost contact or interest during the pandemic } \\
\text { but could not be seen in person }\end{array}$ & $\$ 2.00$ \\
\hline $\mathrm{C}$ & $\begin{array}{l}\text { Remote technical support } \\
\text { package }\end{array}$ & $\begin{array}{l}\text { For participants using their own smartphone } \\
\text { device and experiencing technical difficul- } \\
\text { ties installing or using the app }\end{array}$ & $\begin{array}{l}\text { To support or troubleshoot self-installation } \\
\text { and use of the app }\end{array}$ & $\$ 2.55$ \\
\hline
\end{tabular}


Table 3. Care package contents and methods.

\begin{tabular}{|c|c|c|c|c|}
\hline \multirow[t]{2}{*}{ Function served and package contents and method } & \multicolumn{4}{|c|}{ Package type } \\
\hline & $\mathrm{A}_{0}$ & $\mathrm{~A}_{1}$ & $\mathrm{~B}$ & $\mathrm{C}$ \\
\hline \multicolumn{5}{|l|}{ Delivery } \\
\hline Postal delivery: delivered via United States Postal Service & $\boldsymbol{\nu}^{\mathrm{a}}$ & & & \\
\hline Hand delivery: driven to participant's home and left on doorstep by research team member & & $\boldsymbol{V}$ & $\boldsymbol{V}$ & $\boldsymbol{V}$ \\
\hline Study phone: Samsung Galaxy S9 smartphone with charger and protective case & $\boldsymbol{V}$ & $\boldsymbol{V}$ & & \\
\hline $\begin{array}{l}\text { Getting started guide: documentation with study ID, research and support team contact information, and instructions } \\
\text { on first-time phone and app use }\end{array}$ & $\checkmark$ & $\checkmark$ & & \\
\hline \multicolumn{5}{|l|}{ Support } \\
\hline Technical support contact: IT support phone number added into study phone contacts & $\boldsymbol{V}$ & $\boldsymbol{V}$ & & \\
\hline $\begin{array}{l}\text { Help documentation: link to online help documentation for the app loaded onto study phone; if requested, printed } \\
\text { copy of help documentation was included }\end{array}$ & $\boldsymbol{V}$ & $\boldsymbol{V}$ & & \\
\hline $\begin{array}{l}\text { Video walk-through: link to tutorial video with app usage guidance loaded onto study phone; also accessible } \\
\text { within the app's help section }\end{array}$ & & $\checkmark$ & & \\
\hline $\begin{array}{l}\text { Study phone screen sharing software: Samsung Knox technology management installed on study phones for remote } \\
\text { screen sharing and control, with permission }\end{array}$ & $\boldsymbol{V}$ & $\boldsymbol{V}$ & & \\
\hline $\begin{array}{l}\text { Other screen sharing software: Zoom or TeamViewer used for remote screen sharing and control, with permission, } \\
\text { for participants not using study phones }\end{array}$ & & & & $\boldsymbol{V}$ \\
\hline \multicolumn{5}{|l|}{ Return } \\
\hline Paper System Usability Scale (SUS): hard copy of the SUS & $\boldsymbol{V}$ & $\boldsymbol{\sim}$ & & $\boldsymbol{V}$ \\
\hline Digital SUS: link to online version of the SUS installed on study phones & $\boldsymbol{V}$ & $\boldsymbol{\sim}$ & & \\
\hline Paper usability questionnaire: hard copy of in-depth usability questionnaire & $\boldsymbol{V}$ & $\checkmark$ & & $\boldsymbol{V}$ \\
\hline Digital usability questionnaire: link to online version of in-depth usability questionnaire installed on study phones & $\boldsymbol{V}$ & $\boldsymbol{V}$ & & \\
\hline Return envelope: prestamped, preaddressed return envelope to return completed paper questionnaires & $\boldsymbol{V}$ & $\boldsymbol{V}$ & & $\boldsymbol{V}$ \\
\hline \multicolumn{5}{|l|}{ Other (ie, goodwill and engagement) } \\
\hline Token item: word search puzzle book and pencil & $\boldsymbol{V}$ & $\boldsymbol{v}$ & $\boldsymbol{V}$ & $\boldsymbol{V}$ \\
\hline Appreciation letter: letter of thanks and explanation from research team to participants & & $\boldsymbol{\sim}$ & $\checkmark$ & $\boldsymbol{V}$ \\
\hline
\end{tabular}

${ }^{\mathrm{a}}$ Check marks indicate contents were included in package type.

\section{Delivery}

\section{Overview}

In the beginning of the pandemic, we faced the challenge of providing mobile apps to older adults who (1) were not tech savvy enough to download and learn to use the app on their own and/or (2) did not possess the technology required to use the app. To address these challenges, two specific conditions related to intervention delivery needed to be met:

1. Smartphones needed to be delivered to those who did not have their own.

2. Participants needed to begin using the study app and smartphone.

\section{Smartphone Delivery}

Our need to deliver a physical item (ie, a smartphone) to participants who needed one was the primary reason our team created individual care packages for participants. We initially shipped the packages to participants using the United States Postal Service (USPS). We predicted this would eliminate contact between participants and study personnel, in compliance with institutional pandemic restrictions.

Shipping packages with USPS proved efficient in reducing the time required from Project $\mathrm{C}$ team members, but left many delivery aspects outside the control of the study team or inconvenient for participants. Due to the contents of the packages being of high value, the mail recipient's signature was required by USPS. This not only meant that participants needed to be present at the time of delivery, but it also required face-to-face contact between the participant and the postal worker. Each time a participant could not sign for delivery added at least one day's delay. Shipping the packages also affected the cost per package and the time between package creation and delivery.

In the next iteration of the package, the study team decided to hand-deliver care packages to participants, thus reducing sign-off failures, costs, and delays. This also led to the team priming participants by confirming their availability to receive the package and their correct address. Project $\mathrm{C}$ staff took appropriate disinfecting, distancing, and personal protective 
equipment precautions when preparing and hand-delivering packages.

\section{Initiating and Sustaining App Use}

Prepandemic protocols had study personnel demonstrate how to use the app to participants during an in-person meeting. To familiarize participants with the operation of the smartphone and the steps required to initiate their use of the app, a getting started guide was included in the package. Participants were contacted using their preferred method (ie, email, phone call, or text, as specified during their baseline meeting with the study team).

In addition to package components to facilitate use of the app, we included a token item (ie, a word search puzzle book) and letter of appreciation from the team to show goodwill in difficult times and add something fun to the package. Due to delays between initial recruitment and app delivery as well as stress that was likely being experienced by many participants at the time, we believed these would promote participant engagement with the team and the study.

We experienced difficulties contacting a subset of participants who had agreed to the study in 2019 but had not yet received the app. In an attempt to reach and re-engage those participants, smaller packages containing only the token item and appreciation letter were sent to 2 such participants. This was discontinued in later sprints in favor of more cost- and time-effective methods of reaching out to nonresponsive participants.

\section{Support}

We understood that many participants would require some form of technical support to effectively use the app. We brainstormed many ideas for remote support and over time attempted and refined several. First, we included contact information for study personnel (ie, dedicated technical support email and phone number) in each package. We provided in-app one-tap links and a web link to digital copies of help documentation demonstrating the features of the app and how to access each app function. Paper help documents were provided as needed.

In later iterations of the package, we created a video walk-through and tutorial of the app with voice-over instructions. We determined that seeing the app used on a screen would help some participants learn how to use the app without needing synchronous interactions with study personnel, thus allowing them to use the app at their own pace.

We anticipated that while phone interactions and video tutorials were adequate to address the needs of some participants, there would be others who would benefit from more detailed, personalized support. We implemented screen sharing capabilities to offer such support. Study-provided phones were equipped with the option to view and control the phone screen remotely using Knox Manage Remote Support, which was conducted with explicit permission from the participant when needed. This helped when participants could not effectively explain what was on their screen via a phone call and, thus, was viewed as an "invaluable" feature by study personnel.

Additionally, some participants used their own smartphones and were able to download and install the app following email instructions, but required additional remote support. Initially we attempted to use Zoom, a software platform for web-based meetings that allows for screen sharing. We found that installing, registering for, accessing, and using Zoom was too cumbersome for first-time users or caused problems due to system requirements. The team thereafter tested the use of TeamViewer, a similar platform that permits screen sharing by having a participant read a unique 10-digit "Partner ID" to a study team member, leading to on-screen commands to set up and initiate screen sharing. Although TeamViewer required a paid subscription to control the partner's device, the screen sharing function significantly reduced setup time from over one hour (ie, the time it took for the one attempt to use Zoom) to an average of approximately 10 minutes.

Most privacy concerns about screen sharing were mitigated by openly communicating and building rapport with participants. The screen sharing tools were explained to each participant as well as each step involved in allowing the team member to view their screen. Participants were given opportunities to discuss anything about which they were unsure and it was made clear they could opt out of screen sharing and have technical support delivered solely over the phone instead.

\section{Return}

The simplest viable method was to provide participants with a one-tap link to digital usability assessments installed on the study phone. Online assessments ensured uniform delivery and collection of responses in one location. However, we hypothesized that some participants would not want to complete questionnaires on a phone. Moreover, for those not using a study phone, we initially considered but rejected options such as mailing a printed Quick Response (QR) code to the assessment link or emailing the link. Therefore, we added paper usability assessments to care packages along with a stamped, self-addressed return envelope. We theorized that older adult participants would vary in comfort and ability to complete online questionnaires, but all would be able to return mailed questionnaires.

To increase the return of assessments, the team contacted participants by phone and email, when available. In total, 4 participants remained unreachable after multiple attempts.

\section{Evaluation}

The first of four packages were sent to participants on April 16, 2020, approximately one month from Project $C$ initiation and within the 6-weeks-or-less target. Subsequent deliveries were maintained on the 2-week schedule through June 2020 per our goal; after a delay, package delivery resumed on the 2-week schedule in late July 2020 (see Table 4). The delay was judged as probably unavoidable but constituted a failure to achieve the target pace. 
Table 4. Care package delivery timeline.

\begin{tabular}{llll}
\hline Delivery date & Care package type & Number of packages delivered in batch & Within approximately 2 weeks of prior batch? \\
\hline April 16, 2020 & $\mathrm{A}_{0}$ & 4 & N/A \\
April 30, 2020 & $\mathrm{A}_{1}$ & 2 & Yes \\
April 30, 2020 & $\mathrm{B}$ & 2 & Yes \\
May 14, 2020 & $\mathrm{C}$ & 4 & Yes \\
June 4, 2020 & $\mathrm{A}_{1}$ & 2 & Yes \\
July 23, 2020 & $\mathrm{A}_{1}$ & 1 & No \\
August 5, 2020 & $\mathrm{A}_{1}$ & 6 & Yes \\
\hline
\end{tabular}

${ }^{\mathrm{a}} \mathrm{N} / \mathrm{A}$ : not applicable; this was the first delivery date.

During the 6-month period, 19 participants were invited to complete usability assessments in their care packages. Of these, $10(53 \%)$ returned the short, 10-item SUS. Of those 10 participants, $6(60 \%)$ used the link on the study phone, $2(20 \%)$ were emailed the link after providing an email address, and 2 (20\%) completed the questionnaire by phone after receiving a follow-up phone call. None successfully returned a mailed SUS, though we do not know how many attempted without success, for example, if their response was lost in the mail due to campus reorganization of mail delivery services. Out of 19 participants, $8(42 \%)$ returned the longer, in-depth questionnaire to the study team. Of those, 5 (63\%) used the in-phone link, 2 (25\%) completed by phone, and $1(13 \%)$ both returned a paper version and completed the questionnaire online.

Providing email links or having participants respond to assessment questionnaires over the phone are examples of our iterative process enabling our team to respond and adjust our approach based on the unsolicited feedback of our participants. We did not initially have email addresses for our participants and we anticipated that the use of USPS and paper questionnaires would be utilized more frequently than they were. When participants requested that they be allowed to fill out the questionnaire through email, and provided the team an email address, or indicated they had time to discuss the questionnaire and provide answers over the phone, we were able to adjust to meet their needs. While these changes did not constitute a new care package iteration, they are still agile adjustments that our team was able to make as we gained more information about the preferences of our participants.

\section{Discussion}

\section{Principal Findings}

During the COVID-19 pandemic, some clinical trials were forced to shut down [48], while others found ways to adapt [49]. To adapt technology-based research, we created the FAR Ops framework, based on spaceflight operations and Agile processes. Guided by the framework, we iteratively developed and tested solutions, which enabled us to deliver a study intervention and collect usability feedback from older adults, remotely. We succeeded in designing and deploying a minimum viable product in less than 6 weeks, generally maintained a 2-week sprint cycle, and achieved a $40 \%$ to $50 \%$ return rate for usability assessment instruments.
Our work demonstrates the feasibility of "going remote" to deliver and assess technology interventions to a diverse population during a pandemic and likely in future, less dire circumstances. Researchers and practitioners can replicate our approach as an alternative to face-to-face interactions. Doing so not only permits work to continue during circumstances such as communicable disease outbreaks and natural disasters, but can also save costs, accommodate people with disability or other reasons for staying at home (eg, caregiving responsibilities), reduce travel time and reliance on transportation for participants and staff, allow for testing in natural settings, and overall increase the diversity of participants $[11,12,18,50]$.

The FAR Ops conceptual model provides a framework for such endeavors. Its Agile project management approach can be replicated to ensure goals are met on time, progress is made at a rapid pace, and innovation is embedded in the process by continually refining the solution based on emerging needs and feedback from testing [40]. We demonstrated how simple Agile concepts, such as explicitly setting expectations for a rapid first "good enough" iteration or minimum viable product and short sprint cycles, resulted in rapid, continuous progress.

Our Agile approach also helped the team iteratively respond to new information, which led to adaptations in package design to meet needs, solve problems, and improve effectiveness and efficiency. This is not possible in a traditional or waterfall approach to project management, wherein solution development is front-loaded and prolonged in an effort to come up with the ideal solution, leaving little time, opportunity, and resources available to redesign and retest the solution if it turns out to not be as ideal as assumed. In fact, it has been documented that technologies developed without integrating iterative testing and redesign opportunities result in products that do not effectively respond to user needs [51-53]. Compared to waterfall approaches, Agile allows feedback from customers-in our case, participants - to dictate changes, rather than assuming the design team can predict customer experiences [54]. However, because an Agile approach promotes quickly creating the minimum viable product, this can result in early solutions having fewer features. This was true in our case, as participants in earlier iterations did not receive tutorial videos or screen sharing, leading to challenges. At the same time, these challenges inspired these subsequent features to be added, reinforcing the importance of the Agile approach for innovation and 
determining the contents of the solution based on actual experimentation [55].

We adopted an Agile Mindset, meaning we went beyond specific Agile techniques and even downplayed the need to adopt specific Agile techniques, such as measuring velocity and throughput, diagramming, or having formal scrum teams [56,57]. We emphasized instead the Agile Mindset principle of establishing a psychologically safe culture within which the team was empowered to work autonomously, make quick progress, prefer "good enough" to "perfect," and learn from failures. However, we acknowledge by some standards, the project's specific methods were discordant with textbook Agile approaches.

Much like user-centered design, our approach was iterative, catered to diverse participant needs, and made changes to designs based on evidence provided by users [51-53]. We assert that user-centered design and the Agile approach are complementary - the Agile approach can help operationalize user-centered design best practices, while user-centered design compels solutions that fit the end user. The concept of the Agile approach and user-centered design compatibility is detailed in Holden and Boustani [58] and Holden et al [59].

A significant challenge overcome by our study team was the lack of existing methodologies and guidelines available for remote usability testing with older adults. Recently, McLaughlin et al [8] outlined various tools available for different kinds of remote usability testing of medical devices and recommended that study personnel be available for phone support during tests with older adults. Beyond this advice, there is no clear guidance from human-computer interaction, usability, mHealth, or other literature on how to operationalize a fully remote study protocol for older adult participants. Based on the results of our work, we present additional recommendations for remote operations with diverse populations in Table 5.

Table 5. Recommendations for remote usability studies with diverse populations.

\begin{tabular}{lll}
\hline Recommendation & Details \\
\hline Screen sharing and screen control & $\bullet$ & Valuable for technical support \\
& $\bullet$ & Use alongside phone-based support \\
& $\bullet$ & Allows research personnel to more clearly understand participant challenges \\
& $-\quad$ Use screen sharing platforms that minimize input required from participant (eg, Knox Manage \\
&
\end{tabular}

Phone-based interactions

Email
- Some participants may prefer to be contacted by telephone

- Allows for participants to talk through impressions and responses to usability evaluations

- Can enable researchers to gather more detailed responses to open-ended questions

- Determine if participant would prefer to answer questionnaires over the phone

- Some participants may be very familiar with email interactions while others are not

- Determine if participant has a valid email and would prefer to be emailed a link to a usability questionnaire, over other methods
About $50 \%$ of participants returned their usability assessments, fewer than desired and, thus, limiting the success of the project. This may be due to reduced levels of engagement overall, especially during unusual circumstances (eg, the COVID-19 pandemic), or as a result of remote and often asynchronous communication. Prior work recommends one-on-one, synchronous conversation as the most effective method to elicit information from older adults [27,28] and may explain why some participants preferred to complete assessments by phone, even though this option was not initially offered. Synchronous interactions may have been preferred by participants to provide lengthier or supplemental feedback to the team. Indeed, phone responses were, on average, longer than those through self-administered modes. We further hypothesize that some participants may have had difficulty typing their responses or using online questionnaires, which contributed to nonresponse or preference to complete assessments by phone, though this was not communicated to the study team by any participants. This may have been especially so for the longer questionnaire, although response rates for the shorter structured 10-item SUS $(53 \%)$ and the longer questionnaire with free-text responses (42\%) were not exceptionally different. It is important to note that while this flexibility in data collection methodology enabled us to collect more responses from participants, it also introduced variability in the level of detail of collected responses. This could challenge the fidelity of studies that heavily rely on consistent data collection techniques.

Maintaining participant engagement was difficult for our team and may indicate that it would be valuable if, in the future, we were more closely able to replicate face-to-face interactions when assessing intervention usability. We are currently exploring adapting existing synchronous remote usability methods for older adults.

The ages of the 19 participants who received Type A and C packages were, overall, representative of the larger target group: 12 in their 60s, 5 in their 70s, and 1 participant in their 80s. Younger participants were more likely than older participants to return usability assessments to the study team; of those older participants who did return assessments, they were more likely to prefer phone interactions over electronic responses. The two SUS assessments performed over the phone were collected from participants in their $70 \mathrm{~s}$, while those in their 60 s returned the surveys electronically. Only 1 individual in their 70s completed the longer usability questionnaire, while the rest were returned by younger participants. Although any conclusions are speculative given the small sample size, it may be easier to "go remote" with younger older adults. 
A limitation of this study is that it was not designed to compare our approach with others, prohibiting conclusions about its superiority. We also did not design our study to compare various package iterations to each other. During the project, decisions on design iterations were based on relatively small samples of participant feedback and impressions of study personnel. We report only 6 months of the project. Our sample was relatively diverse, but overrepresented younger, White, and female individuals. We believe that limitations with our sample are reasonable due to the nature of the COVID-19 pandemic. We did not formally assess the team's fidelity to Agile principles or use certain Agile formalisms, such as velocity or control charting, to track progress.

\section{Conclusions}

Combining a spaceflight model with an Agile approach allowed our team to adopt remote operations for our mHealth trial, in response to interruptions from the COVID-19 pandemic. Our approach can be useful for other research or practical projects

under similar circumstances or to improve efficiency, cost, effectiveness, and participant diversity in general. Indeed, although the COVID-19 pandemic was the impetus for "going remote," we believe further iterating remote operations for older adults will result in these approaches being preferable to traditional ones for many reasons.

In addition to offering a replicable approach, this paper tells the often-untold story of practical challenges faced by mHealth projects and practical strategies used, as another article in this journal [45] compels us to do:

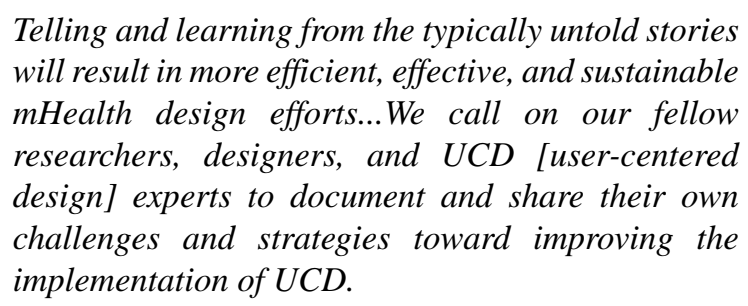

\section{Acknowledgments}

We thank members of the Brain Safety Lab for their input. The authors of this paper were supported by grant R01AG056926 from the National Institute on Aging of the National Institutes of Health (NIH). The content is solely the responsibility of the authors and does not necessarily represent the official views of NIH.

\section{Authors' Contributions}

JRH and RJH conceived this paper. All authors wrote and edited the paper. All authors approved the final version.

\section{Conflicts of Interest}

None declared.

\section{References}

1. Messenger JC. Telework in the 21st Century: An Evolutionary Perspective. Northampton, MA: Edward Elgar Publishing, Inc; Nov 29, 2019.

2. HHS issues new report highlighting dramatic trends in Medicare beneficiary telehealth utilization amid COVID-19. US Department of Health \& Human Services. 2020 Jul 28. URL: https://www.hhs.gov/about/news/2020/07/28/ hhs-issues-new-report-highlighting-dramatic-trends-in-medicare-beneficiary-telehealth-utilization-amid-covid-19.html [accessed 2021-02-25]

3. Loeb AE, Rao SS, Ficke JR, Morris CD, Riley LH, Levin AS. Departmental experience and lessons learned with accelerated introduction of telemedicine during the COVID-19 crisis. J Am Acad Orthop Surg 2020;28(11):e469-e476. [doi: 10.5435/jaaos-d-20-00380]

4. Andrienko O. Semrush Blog. 2020 Apr 20. URL: https://www.semrush.com/blog/ecommerce-covid-19/ [accessed 2021-02-25]

5. Lai B, Chiu C, Pounds E, Tracy T, Mehta T, Young H, et al. COVID-19 modifications for remote teleassessment and teletraining of a complementary alternative medicine intervention for people with multiple sclerosis: Protocol for a randomized controlled trial. JMIR Res Protoc 2020 Jul 03;9(7):e18415 [FREE Full text] [doi: 10.2196/18415] [Medline: 32540838]

6. DeMatthews D, Knight D, Reyes P, Benedict A, Callahan R. From the field: Education research during a pandemic. Educ Res 2020 Jul 07;49(6):398-402. [doi: 10.3102/0013189x20938761]

7. Omary MB, Eswaraka JR, Kimball SD, Moghe PV, Panettieri RA, Scotto KW. The COVID-19 pandemic and research shutdown: Staying safe and productive. J Clin Invest 2020 Jun 01;130(6):2745-2748 [FREE Full text] [doi: 10.1172/JCI138646] [Medline: 32243259]

8. McLaughlin AC, DeLucia PR, Drews FA, Vaughn-Cooke M, Kumar A, Nesbitt RR, et al. Evaluating medical devices remotely: Current methods and potential innovations. Hum Factors 2020 Nov;62(7):1041-1060 [FREE Full text] [doi: 10.1177/0018720820953644] [Medline: 32960093 ]

9. Hartson HR, Castillo JC. Remote evaluation for post-deployment usability improvement. In: Proceedings of the Working Conference on Advanced Visual Interfaces (AVI '98). New York, NY: Association for Computing Machinery; 1998 May Presented at: Working Conference on Advanced Visual Interfaces (AVI '98); May 24-27, 1998; L'Aquila, Italy p. $22-29$ URL: https://static.aminer.org/pdf/PDF/000/061/037/remote evaluation for post deployment usability improvement.pdf [doi: 10.1145/948496.948499] 
10. Castillo JC, Hartson HR, Hix DH. Remote usability evaluation: Can users report their own critical incidents? In: Proceedings of the Conference on Human Factors and Computing Systems (CHI '98). New York, NY: Association for Computing Machinery; 1998 Apr Presented at: Conference on Human Factors and Computing Systems (CHI '98); April 18-23, 1998; Los Angeles, CA p. 253-254. [doi: 10.1145/286498.286736]

11. Andreasen M, Nielsen H, Schrøder S, Stage J. What happened to remote usability testing? An empirical study of three methods. In: Proceedings of the SIGCHI Conference on Human Factors in Computing Systems (CHI '07). New York, NY: Association for Computing Machinery; 2007 Apr Presented at: SIGCHI Conference on Human Factors in Computing Systems (CHI '07); April 28-May 3, 2007; San Jose, CA p. 1405-1414. [doi: 10.1145/1240624.1240838]

12. Hammontree M, Weiler P, Nayak N. Remote usability testing. Interactions 1994 Jul;1(3):21-25. [doi: 10.1145/182966.182969]

13. Tullis T, Fleischman S, Mcnulty M, Cianchette C, Bergel M. An empirical comparison of lab and remote usability testing of web sites. In: Proceedings of the 11th Usability Professionals Association Conference. 2002 Jul Presented at: 11th Usability Professionals Association Conference; July 8-12, 2002; Orlando, FL URL: https://citeseerx.ist.psu.edu/viewdoc/ download?doi=10.1.1.457.3080\&rep=rep1\&type $=$ pdf

14. Bernheim Brush AJ, Ames M, Davis J. A comparison of synchronous remote and local usability studies for an expert interface. In: Proceedings of the Conference on Human Factors in Computing Systems (CHI04). New York, NY: Association for Computing Machinery; 2004 Apr Presented at: Conference on Human Factors in Computing Systems (CHI04); April 24-29, 2004; Vienna, Austria p. 1179-1182. [doi: 10.1145/985921.986018]

15. Diamantidis CJ, Ginsberg JS, Yoffe M, Lucas L, Prakash D, Aggarwal S, et al. Remote usability testing and satisfaction with a mobile health medication inquiry system in CKD. Clin J Am Soc Nephrol 2015 Aug 07;10(8):1364-1370 [FREE Full text] [doi: 10.2215/CJN.12591214] [Medline: 26220816]

16. Bruun A, Gull P, Hofmeister L, Stage J. Let your users do the testing: A comparison of three remote asynchronous usability testing methods. In: Proceedings of the SIGCHI Conference on Human Factors in Computing Systems (CHI '09). New York, NY: Association for Computing Machinery; 2009 Apr Presented at: SIGCHI Conference on Human Factors in Computing Systems (CHI '09); April 4-9, 2009; Boston MA p. 1619-1628. [doi: 10.1145/1518701.1518948]

17. Fidas C, Katsanos C, Papachristos E, Tselios N, Avouris N. Remote usability evaluation methods and tools: A survey. In: Proceedings of the 11th Panhellenic Conference on Informatics (PCI 2007). 2007 Presented at: 11th Panhellenic Conference on Informatics (PCI 2007); May 18-20, 2007; Patras, Greece p. 151-162 URL: http://citeseerx.ist.psu.edu/viewdoc/ download?doi=10.1.1.545.7719\&rep=rep1\&type=pdf

18. Petrie H, Hamilton F, King N, Pavan P. Remote usability evaluations with disabled people. In: Proceedings of the SIGCHI Conference on Human Factors in Computing Systems (CHI '06). New York, NY: Association for Computing Machinery; 2006 Apr Presented at: SIGCHI Conference on Human Factors in Computing Systems (CHI '06); April 22-27, 2006; Montréal, QC p. 1133-1141. [doi: 10.1145/1124772.1124942]

19. Anderson M, Perrin A. Tech adoption climbs among older adults. Pew Research Center. Washington, DC: Pew Research Center; 2017 May 17. URL: https://www.pewresearch.org/internet/2017/05/17/tech-adoption-climbs-among-older-adults/ [accessed 2021-02-25]

20. Wildenbos GA, Peute L, Jaspers M. Aging barriers influencing mobile health usability for older adults: A literature based framework (MOLD-US). Int J Med Inform 2018 Jun;114:66-75. [doi: 10.1016/j.ijmedinf.2018.03.012] [Medline: 29673606]

21. Ramsetty A, Adams C. Impact of the digital divide in the age of COVID-19. J Am Med Inform Assoc 2020 Jul 01;27(7):1147-1148 [FREE Full text] [doi: 10.1093/jamia/ocaa078] [Medline: $\underline{\text { 32343813] }}$

22. Internet/broadband fact sheet. Pew Research Center. Washington, DC: Pew Research Center; 2019 Jun 12. URL: https:/ /www.pewresearch.org/internet/fact-sheet/internet-broadband/ [accessed 2021-02-25]

23. Choi NG, Dinitto DM. The digital divide among low-income homebound older adults: Internet use patterns, eHealth literacy, and attitudes toward computer/internet use. J Med Internet Res 2013 May 02;15(5):e93 [FREE Full text] [doi: 10.2196/jmir.2645] [Medline: 23639979]

24. Mitchell U, Chebli P, Ruggiero L, Muramatsu N. The digital divide in health-related technology use: The significance of race/ethnicity. Gerontologist 2019 Jan 09;59(1):6-14. [doi: 10.1093/geront/gny138] [Medline: 30452660]

25. Smith A. Older adults and technology use. Pew Research Center. Washington, DC: Pew Research Center; 2014 Apr 03. URL: https://www.pewresearch.org/internet/2014/04/03/older-adults-and-technology-use/ [accessed 2021-02-25]

26. Levy H, Janke AT, Langa KM. Health literacy and the digital divide among older Americans. J Gen Intern Med 2015 Mar;30(3):284-289 [FREE Full text] [doi: 10.1007/s11606-014-3069-5] [Medline: 25387437]

27. Dickinson A, Arnott J, Prior S. Methods for human - Computer interaction research with older people. Behav Inf Technol 2007 Jul;26(4):343-352. [doi: 10.1080/01449290601176948]

28. Franz R, Munteanu C, Barbosa NB, Baecker R. Time to retire old methodologies? Reflecting on conducting usability evaluations with older adults. In: Proceedings of the 17th International Conference on Human-Computer Interaction with Mobile Devices and Services Adjunct (MobileHCI '15). New York, NY: Association for Computing Machinery; 2015 Aug Presented at: 17th International Conference on Human-Computer Interaction with Mobile Devices and Services Adjunct (MobileHCI '15); August 24-27, 2015; Copenhagen, Denmark p. 912-915. [doi: 10.1145/2786567.2794303]

29. Silva P, Nunes F. 3 x 7 usability testing guidelines for older adults. In: Proceedings of the 3rd Mexican Workshop on Human Computer Interaction (MexIHC '10). San Luis Potosí, Mexico: Universidad Politécnica de San Luis Potosí; 2010 Nov 
Presented at: 3rd Mexican Workshop on Human Computer Interaction (MexIHC '10); November 8-10, 2010; San Luis Potosí, Mexico p. 1-8 URL: http://mural.maynoothuniversity.ie/6030/1/PS-Usability-Testing.pdf

30. Abebe E, Campbell NL, Clark DO, Tu W, Hill JR, Harrington AB, et al. Reducing anticholinergic medication exposure among older adults using consumer technology: Protocol for a randomized clinical trial. Res Soc Adm Pharm 2020 Oct: 1 (forthcoming). [doi: 10.1016/j.sapharm.2020.10.010]

31. Holden RJ, Campbell NL, Abebe E, Clark DO, Ferguson D, Bodke K, Brain Health Patient Safety Laboratory. Usability and feasibility of consumer-facing technology to reduce unsafe medication use by older adults. Res Social Adm Pharm 2020 Jan;16(1):54-61 [FREE Full text] [doi: 10.1016/j.sapharm.2019.02.011] [Medline: 30853507]

32. Howell E. A brief history of Mars missions. Space. 2019. URL: https://www.space.com/13558-historic-mars-missions.html [accessed 2021-02-25]

33. Humans in space overview. National Aeronautics and Space Administration (NASA). 2018 Jul 25. URL: https://www. nasa.gov/topics/humans-in-space/overview [accessed 2021-02-25]

34. Missions. Jet Propulsion Laboratory, NASA. URL: https://www.jpl.nasa.gov/missions/?type=current [accessed 2021-02-25]

35. Mishkin A. Sojourner: An Insider's View of the Mars Pathfinder Mission. New York, NY: The Berkley Publishing Group; 2003.

36. Woolford B, Sipes W, Fiedler E. Human space flight. In: Salvendy G, editor. Handbook of Human Factors and Ergonomics. Fourth edition. Hoboken, NJ: John Wiley \& Sons, Inc; 2012:910-927.

37. Bell E, Coan D, Oswald D. A discussion on the making of an EVA: What it really takes to walk in space. In: Proceedings of the SpaceOps 2006 Conference. 2006 Presented at: SpaceOps 2006 Conference; June 19-23, 2006; Rome, Italy p. 1-20 URL: https://arc.aiaa.org/doi/pdf/10.2514/6.2006-5570 [doi: 10.2514/6.2006-5570]

38. Heiney A. SpaceX-3 science payloads return to Kennedy. National Aeronautics and Space Administration (NASA). 2014 May 23. URL: https://www.nasa.gov/content/spacex-3-science-payloads-return-to-kennedy [accessed 2021-02-25]

39. Dove R. Knowledge management, response ability, and the agile enterprise. J Knowl Manag 1999 Mar;3(1):18-35. [doi: $10.1108 / 13673279910259367]$

40. Beck K, Beedle M, van Bennekum A, Cockburn A, Cunningham W, Fowler M, et al. Manifesto for agile software development. Agile Manifesto. 2001. URL: https://agilemanifesto.org/ [accessed 2021-02-25]

41. Boustani MA, van der Marck MA, Adams N, Azar JM, Holden RJ, Vollmar HC, et al. Developing the agile implementation playbook for integrating evidence-based health care services into clinical practice. Acad Med 2019;94(4):556-561. [doi: 10.1097/acm.0000000000002497]

42. Holden RJ, Boustani MA. The value of an "agile" mindset in times of crisis. Modern Healthcare. 2020 May 11. URL: https:/ /Www.modernhealthcare.com/opinion-editorial/value-agile-mindset-times-crisis [accessed 2021-02-25]

43. Callahan CM, Unverzagt FW, Hui SL, Perkins AJ, Hendrie HC. Six-item screener to identify cognitive impairment among potential subjects for clinical research. Med Care 2002 Sep;40(9):771-781. [doi: 10.1097/00005650-200209000-00007] [Medline: 12218768]

44. Holden RJ, Bodke K, Tambe R, Comer RS, Clark DO, Boustani M. Proceedings of the International Symposium on Human Factors and Ergonomics in Health Care. 2016 Jul 22 Presented at: International Symposium on Human Factors and Ergonomics in Health Care; April 13-16, 2016; San Diego, CA p. 25-27. [doi: 10.1177/2327857916051003]

45. Cornet VP, Toscos T, Bolchini D, Rohani Ghahari R, Ahmed R, Daley C, et al. Untold stories in user-centered design of mobile health: Practical challenges and strategies learned from the design and evaluation of an app for older adults with heart failure. JMIR Mhealth Uhealth 2020 Jul 21;8(7):e17703 [FREE Full text] [doi: 10.2196/17703] [Medline: 32706745]

46. Srinivas P, Cornet V, Holden R. Human factors analysis, design, and evaluation of Engage, a consumer health IT application for geriatric heart failure self-care. Int J Hum Comput Interact 2017;33(4):298-312 [FREE Full text] [doi: 10.1080/10447318.2016.1265784] [Medline: 30429638]

47. Holden RJ. A Simplified System Usability Scale (SUS) for Cognitively Impaired and Older Adults. In: Proceedings of the International Symposium on Human Factors and Ergonomics in Health Care. 2020 Sep 16 Presented at: International Symposium on Human Factors and Ergonomics in Health Care; March 8-11, 2020; Toronto, ON p. 180-182. [doi: $10.1177 / 2327857920091021]$

48. Thornton J. Clinical trials suspended in UK to prioritise COVID-19 studies and free up staff. BMJ 2020 Mar 23;368:m1172. [doi: 10.1136/bmj.m1172] [Medline: 32205354]

49. Verna EC, Serper M, Chu J, Corey K, Fix OK, Hoyt K, et al. Clinical research in hepatology in the COVID-19 pandemic and post-pandemic era: Challenges and the need for innovation. Hepatology 2020 Nov;72(5):1819-1837 [FREE Full text] [doi: 10.1002/hep.31491] [Medline: 32740969]

50. Dray S, Siegel D. Remote possibilities?: International usability testing at a distance. Interactions 2004 Mar;11(2):10-17. [doi: $10.1145 / 971258.971264]$

51. Goldberg L, Lide B, Lowry S, Massett HA, O'Connell T, Preece J, et al. Usability and accessibility in consumer health informatics current trends and future challenges. Am J Prev Med 2011 May;40(5 Suppl 2):S187-S197. [doi: 10.1016/j.amepre.2011.01.009] [Medline: 21521594] 
52. Marquard JL, Zayas-Cabán T. Commercial off-the-shelf consumer health informatics interventions: Recommendations for their design, evaluation and redesign. J Am Med Inform Assoc 2012;19(1):137-142 [FREE Full text] [doi: 10.1136/amiajnl-2011-000338] [Medline: 21727206]

53. Zayas-Cabán T, Dixon BE. Considerations for the design of safe and effective consumer health IT applications in the home. Qual Saf Health Care 2010 Oct;19 Suppl 3:i61-i67. [doi: 10.1136/qshc.2010.041897] [Medline: 20959321]

54. VanderLeest S, Buter A. Escape the waterfall: Agile for aerospace. In: Proceedings of the 2009 IEEE/AIAA 28th Digital Avionics Systems Conference. 2009 Presented at: 2009 IEEE/AIAA 28th Digital Avionics Systems Conference; October 23-29, 2009; Orlando, FL p. 6.D.3-1-6.D.3-16. [doi: 10.1109/dasc.2009.5347438]

55. Boustani M, Holden R, Azar J, Solid C. The Agile Network: A Model to Foster Innovation, Implementation, and Diffusion in Healthcare Systems. St Paul, MN: Beaver's Pond Press; 2019.

56. Radigan D. Five agile metrics you won't hate. Atlassian Agile Coach. URL: https://www.atlassian.com/agile/ project-management/metrics [accessed 2021-02-25]

57. Chowdhury A. Agile metrics: The 15 that actually matter for success. Plutora. 2020. URL: https://www.plutora.com/blog/ agile-metrics [accessed 2021-02-25]

58. Holden RJ, Boustani MA. Design and usability methods: Agile innovation and evaluation of interventions for patients and families. In: Holden RJ, Valdez RS, editors. The Patient Factor: Theories and Methods for Patient Ergonomics. Boca Raton, FL: CRC Press; 2021.

59. Holden RJ, Boustani MA, Azar J. Agile innovation to transform healthcare: Innovating in complex adaptive systems is an everyday process, not a light bulb event. BMJ Innov 2021 Jan 28:1-7 [FREE Full text] [doi: 10.1136/bmjinnov-2020-000574]

\author{
Abbreviations \\ FAR Ops: Framework for Agile and Remote Operations \\ HIPAA: Health Insurance Portability and Accountability Act \\ IRB: Institutional Review Board \\ mHealth: mobile health \\ NIH: National Institutes of Health \\ PI: principal investigator \\ Project C: Project COVID \\ QR: Quick Response \\ SUS: System Usability Scale \\ UCD: user-centered design \\ USPS: United States Postal Service
}

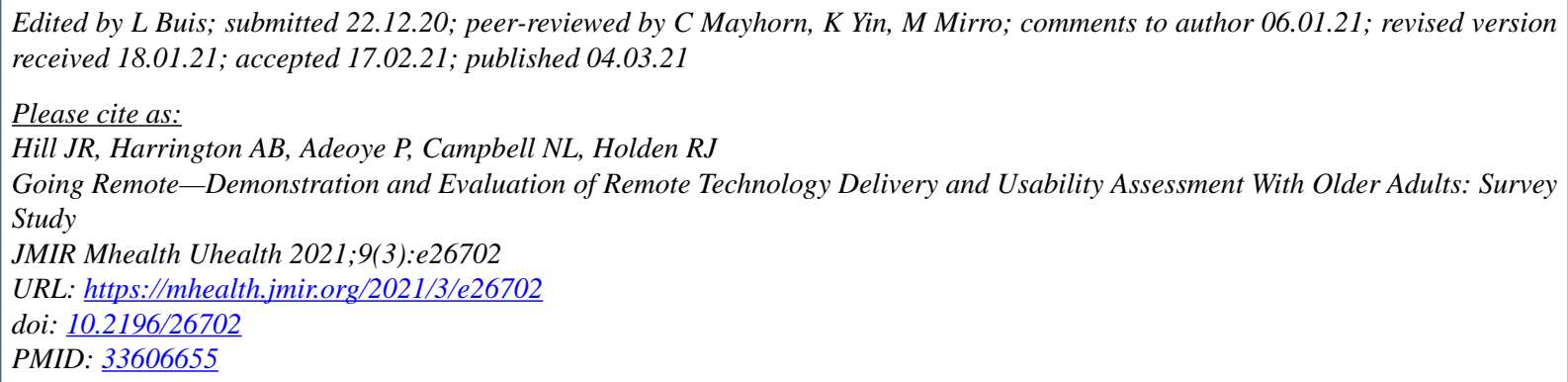

CJordan R Hill, Addison B Harrington, Philip Adeoye, Noll L Campbell, Richard J Holden. Originally published in JMIR mHealth and uHealth (http://mhealth.jmir.org), 04.03.2021. This is an open-access article distributed under the terms of the Creative Commons Attribution License (https://creativecommons.org/licenses/by/4.0/), which permits unrestricted use, distribution, and reproduction in any medium, provided the original work, first published in JMIR mHealth and uHealth, is properly cited. The complete bibliographic information, a link to the original publication on http://mhealth.jmir.org/, as well as this copyright and license information must be included. 